\title{
Charm production at HERA, proton structure, the charm mass, and Higgs Yukawa couplings
}

\author{
Achim Geiser* \\ DESY Hamburg \\ E-mail: Achim.Geiser@desy.de
}

Charm production at HERA (H1 and ZEUS) and its embedding into the Standard Model in the context of results from other experiments are discussed. The latest results on charm production at HERA ( $D^{*}$ and inclusive cross sections, fragmentation fractions, $\psi^{\prime}$ vs. $J / \psi$ ) and their impact on measurements of the proton structure are summarised. Related measurements of the charm quark mass and a determination of its QCD running are presented. Finally, measurements of the charm, beauty and top quark masses from HERA, LEP and LHC are reinterpreted in terms of the corresponding Higgs Yukawa couplings. A graphical representation of the running of the Yukawa couplings derived from measurements is obtained for the first time.

VIII International Workshop On Charm Physics

5-9 September, 2016

Bologna, Italy

${ }^{*}$ Speaker. 


\section{Introduction}

The charm quark is one of the fundamental ingredients of the Standard Model, and directly appears in the Standard Model Lagrangian. Measurements of charm quark production and its properties therefore have a strong impact on our understanding of the Standard Model. This contribution focuses on recent measurements, in particular of charm quark production at the HERA collider by the $\mathrm{H} 1$ and ZEUS experiments, and on the relation of these measurements to other recent measurements and their interpretation. Measurements of the charm quark mass and its running in the context of QCD are presented and discussed. These measurements, together with corresponding measurements of the beauty quark mass at LEP and HERA and top quark masses at LHC, are then reinterpreted in the context of the full Standard Model, in which masses are represented by the corresponding more fundamental Higgs Yukawa couplings. The status of the experimental evidence for the running of these couplings is worked out and graphically represented for the first time, including a comparison to the Yukawa couplings directly measured at LHC. Figures are included in this writeup only for results which are not yet finally published.

\section{Recent measurements of charm production at HERA}

A review of all measurements related to open charm production at HERA up to 2015 can be found in [1], with some extensions in [2]. A review of charmonium production can be found in [3], and of diffractive charm production in [4]. Here we will focus on the most recently published HERA charm measurements.

The final cross sections for differential $D^{*}$ production in Deep Inelastic Scattering (DIS) from H1 and ZEUS were combined [5] and compared to next-to-leading order (NLO) QCD predictions. Differential cross sections in quantities like transverse momentum $\left(p_{T}\right)$, pseudorapidity $(\eta)$ or fractional energy flow $(z)$ were obtained with ultimate HERA precision. Their advantage with respect to cross sections depending only on inclusive quantities [6] like photon virtuality $\left(Q^{2}\right)$ and inelasticity $(y)$ is the sensitivity to kinematic details of charm production not accessible otherwise. Their disadvantage is the larger uncertainty of the corresponding NLO theory predictions, typically 3-5 times larger than the one of the data. It is demonstrated that within these large uncertainties it is possible to find a fixed set of theory parameters with which all data distributions can be simultaneously described using experimental uncertainties only. The conclusion [5] is that the data are described very reasonably, but that more work is needed on the theory side (e.g. NNLO, improved fragmentation) to reduce the uncertainties to a level competitive with the data.

Measurements of the fragmentation fractions of charm quarks to different charm hadron final states at HERA have been compared to and combined [7] with measurements from LEP/SLC, B factories and LHC. The HERA measurements, especially those in photoproduction [8], are very competitive with the other measurements, and substantially contribute to the world average. The good agreement between the measurements from different types of collisions in a large range of energies confirms the universality of fragmentation.

In a ZEUS measurement [9] of the ratio of the exclusive production of $\psi^{\prime}$ and $J / \psi$ mesons in DIS, $e p \rightarrow e p \psi$, many experimental and systematic uncertainties cancel. The measurement of the dependence of this ratio on $Q^{2}$ is in good agreement with earlier measurements by H1 [10], with 
significantly improved precision. It is also consistent with an $\mathrm{H} 1$ measurement in photoproduction [11]. The observed rise of this ratio with $Q^{2}$ is qualitatively consistent with theoretical models based on different assumptions about the $\psi$ wave functions, and starts to quantitatively discriminate between them [9].

\section{Charm and proton structure}

Various fits of the proton parton density functions (PDF) at both NLO and NNLO were obtained by $\mathrm{H} 1$ and ZEUS from the recent final combination of the $\mathrm{H} 1$ and ZEUS inclusive DIS data [12]. All these HERA-data-only fits use values of the charm mass constrained by the combined inclusive HERA charm data [6]. One of the fit variants also explicitly includes these charm data (at NLO), as well as HERA jet data. Good consistency [12] is observed between all these fits, and with various fits obtained by global PDF fitting groups. The inclusive HERA charm data [6] contribute to the reduction of the PDF uncertainties, mainly by constraining the charm quark mass. They are a standard ingredient of all recent global PDF fits [13, 14, 15, 16, 17].

The same inclusive HERA charm data have also been used [18] in conjunction with LHCb charm data [19] and corresponding beauty data [20,21] to constrain the NLO gluon PDF at very low $x$. In particular the LHCb charm data explicitly constrain the gluon distribution down to fractional momenta of order few $10^{-6}$, extending the low $x$ reach by more than an order of magnitude with good precision. Other analyses $[22,23]$ have subsequently obtained similar results. These results are particularly relevant in the context of predictions [22, 24, 25] of the prompt contribution to the cosmic ray neutrino spectrum, as measured e.g. by Ice Cube [26].

\section{Measurements of the charm quark mass and its running}

As mentioned earlier, the inclusive (or differential) HERA charm data in DIS allow one to constrain the charm quark mass and hence to measure its value within different QCD factorization schemes. The cleanest (in the sense of no extra parameters in the QCD matrix elements beyond those appearing in the QCD Lagrangian) such measurements are obtained [27, 6, 28, 29] in the fixed flavour number scheme, and using the so-called $\overline{M S}$ mass $m_{c}\left(m_{c}\right)$ (Table 1). For the latter, the cross section has better perturbative convergence behaviour [30] than using the pole mass. Measurements have been obtained both at NLO and approximate NNLO. Similar measurements using the S-ACOT [31] and FONLL C [29] variable flavour number schemes, both of which have one extra parameter in the merging of the massless and massive parts of the calculation, have also been obtained (Table 1). The latter treats the light flavour contributions at NNLO, and the massive part of the heavy flavour contributions at NLO. Although all use the same HERA charm data [6], the measurements partially differ in the choice of input data sets used for the PDF part of the fit, since charm mass and PDFs are fitted simultaneously. The charm mass can also be fitted simultaneously with the strong coupling constant $\alpha_{s}$ [32]. Preliminary even more precise results are being obtained by adding more data [2,33] and/or improving the NNLO approximation [34].

All these measurements are consistent with each other, and consistent with the world average [35] $1.27 \pm 0.03 \mathrm{GeV}$, obtained mainly from lattice calculations and $e^{+} e^{-}$data with time-like kine- 


\begin{tabular}{|c|c|}
\hline scheme & $m_{c}\left(m_{c}\right)[\mathrm{GeV}]$ \\
\hline FONLL [29] & $1.335 \pm 0.043(\exp )_{-0.000}^{+0.019}(\text { param })_{-0.008}^{+0.011}(\mathrm{mod})_{-0.008}^{+0.033}($ th $)$ \\
\hline FFN [29] & $1.318 \pm 0.054(\exp )_{-0.010}^{+0.011}(\text { param })_{-0.019}^{+0.015}(\mathrm{mod})_{-0.004}^{+0.045}($ th $)$ \\
\hline FFN (HERA) [6] & $1.26 \pm 0.05(\mathrm{exp}) \pm 0.03(\mathrm{mod}) \pm 0.02($ param $) \pm 0.02\left(\alpha_{s}\right)$ \\
\hline FFN (Alekhin et al.) [28] & $\begin{array}{l}1.24 \pm 0.03(\exp )_{-0.02}^{+0.03}(\text { scale })_{-0.07}^{+0.00}(\text { th })(\text { approx. NNLO }) \\
1.15 \pm 0.04(\exp )_{-0.00}^{+0.04}(\text { scale })(\text { NLO })\end{array}$ \\
\hline FFN (Alekhin et al.) [34] & $1.252 \pm 0.018(\exp ) \pm 0.025($ scale $) \pm 0.010($ th $)($ approx. NNLO $)$ \\
\hline$\overline{\mathrm{S}-\mathrm{ACOT}-\chi(\mathrm{CT} 10)[31]}$ & $\begin{array}{l}\left.1.12_{-0.11}^{+0.05} \text { (strategy } 1\right) \\
\left.1.18_{-0.11}^{+0.05} \text { (strategy } 2\right) \\
1.19_{-0.15}^{+0.06}(\text { strategy } 3) \\
1.24_{-0.15}^{+0.06}(\text { strategy } 4) \\
\end{array}$ \\
\hline World average [35] & $1.27 \pm 0.03$ \\
\hline
\end{tabular}

Table 1: List of recent determinations of $m_{c}\left(m_{c}\right)$ from fits to DIS data adjusted from Ref. [29]. The updated 2016 PDG world average value is also reported for reference.

matics. This demonstrates the consistency of the different QCD approaches, and the usefulness of the HERA charm data to perform such measurements.

One of these measurements [6] has been refined further in order to extract information about the charm mass running. In $\mathrm{QCD}$, the $\overline{M S}$ running mass varies (runs) with scale $\mu$, conceptually similar to the running of $\alpha_{s}$. All the measurements above were expressed in terms of the charm quark mass $m_{c}(\mu)$ at the reference scale $\mu=m_{c}$ itself, as is customary, just like $\alpha_{s}$ is often expressed at the scale of the $Z$ boson mass $\alpha_{s}\left(M_{Z}\right)$, by convention. The running of $\alpha_{s}$ is usually studied by separately evaluating it for subprocesses measured at different scales $\mu$ (e.g. $e^{+} e^{-}$center of mass or jet $\left.p_{T}\right)$. Here, the charm data set [6] is subdivided into several subsets, centered on different scales $\mu=\sqrt{Q^{2}+m_{c}^{2}}$. Each of these subsets is then fitted separately, using PDFs refitted from inclusive data only with the appropriate charm mass setting in a way that minimises the very small residual dependence of the charm mass fit on charm effects in the PDF fit [36, 37]. This results in the graphical representation $[36,1]$ of the charm mass running shown in Fig. 1. The running is consistent with QCD expectations.

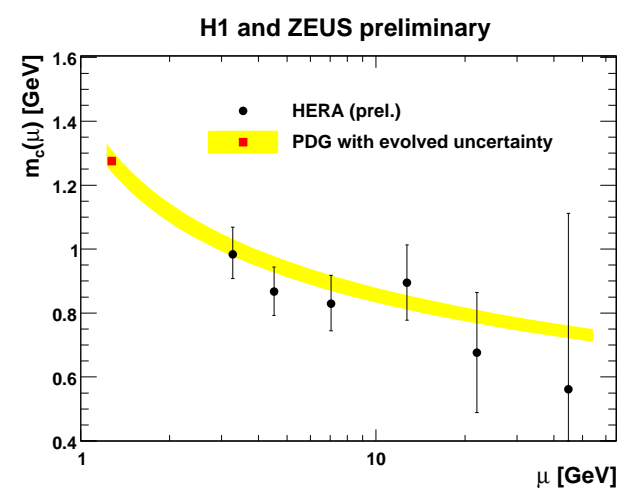

Figure 1: Measured charm mass $m_{c}(\mu)$ in the $\overline{M S}$ running mass scheme as a function of the scale $\mu$ as defined in the text (black points). The red point at scale $m_{c}$ is the PDG world average [38] and the band is its expected one-loop running [39]. 


\section{Running of Higgs Yukawa couplings}

As is well known, in pure QCD, a quark mass term can be introduced as a fundamental contribution to the Lagrangian. In the Standard Model, due to left-right symmetry breaking, such a term violates electroweak gauge invariance. Therefore it has to be 'mimicked' by replacing it with a term coupling to the Higgs field, with a nonzero vacuum expectation value $v=246.22 \mathrm{GeV}$. The 'constant' part of this coupling is then equivalent to a mass term with

$$
m_{q}:=\frac{1}{\sqrt{2}} y_{q} v
$$

where $y_{q}$ is the Higgs Yukawa coupling for the quarks $q=c, b, t$ which we will consider here. The goal is the extraction of a graphical representation of the running of these Yukawa couplings as a function of scale, based on measurements.

The details of how the masses and couplings evolve with scale are scheme dependent. Here, we use the $\overline{M S}$ scheme for QCD at NLO as in the previous section, and a Higgs/electroweak scheme in which Eq. (1) is taken to be universally valid. In the following, all relevant Higgs and electroweak effects are treated to leading (0-loop) order. Thus, in this scheme, the running of the Yukawa couplings is equivalent to the running of the $\overline{M S}$ mass in QCD.

The direct measurements of the Yukawa couplings for top and beauty from Higgs production and decay at the LHC [40] and at the Tevatron [41] are assumed to be obtained at scale $m_{H}$. The QCD $\overline{M S}$ masses which we will use here are all evaluated from real quark pair production cross sections and are assumed to be obtained at scale $\sqrt{Q^{2}+4 m_{c}^{2}}$ for charm at HERA [6, 36, 37], at scale $\sqrt{Q^{2}+4 m_{b}^{2}} \simeq 2 m_{b}$ (cross section dominated by low $Q^{2}$ ) for beauty at HERA [20, 37], at scale $m_{Z}$ for beauty at LEP/SLC [42], and at scale $m_{t \bar{t}}$ for top at LHC [43].

For charm and beauty, Eq. (1), reformulated as $y_{q}=\sqrt{2} \frac{m_{q}}{v}$, can be directly applied to the measurements of $m_{q}(\mu)$ cited above. For $q=c$, they are exactly the ones explained in the previous section. The measurements for $q=b$ are treated similarly.

A theoretically well-defined experimental scale-dependent evaluation of the running of the $\overline{M S}$ top quark mass can not yet be performed from existing published data. Here, we will apply a preliminary approximate procedure to nevertheless extract such an evaluation, which is on much less solid grounds than those for charm and beauty. It should be replaced by a better-defined procedure as soon as corresponding data become available.

We start from a scale depedendent evaluation [43] of the so-called 'MC mass' (here technically the 'hybrid mass') as a function of the $t \bar{t}$ invariant mass $m_{t \bar{t}}$ performed by CMS. Note that the MC mass is mainly extracted from kinematical quantities in the data. The measurements at different $m_{t \bar{t}}$ are statistically (but not necessarily systematically) independent. This was originally used to demonstrate that, within uncertainties and as expected, the MC masses measured at different scales with different kinematics are consistent with a single value [43].

The top pole mass has been extracted by both ATLAS [44] and CMS [45, 46] as a single value from top cross section measurements, for which the data sets partially overlap with those from which the MC mass is extracted. We note that the top pole and MC masses evaluated from LHC data are numerically almost identical, as to be expected [33]. The theoretical uncertainties of this conversion are poorly known, but can be estimated to be of order $1 \mathrm{GeV}$ or less [38, 33]. 
The conversion factor from MC mass to pole mass is therefore very close to 1 , with an estimated uncertainty of less than $1 \%$.

A comparison of the measured top cross section as a function of $m_{t \bar{t}}$ [47] with theoretical predictions at leading order plus parton shower, which use the MC mass, and at NLO, which use the pole mass, shows that all three are reasonably consistent. They are also consistent [48] between ATLAS and CMS and with NNLO predictions. This raises the hope (but does not prove) that, if evaluated as a function of $m_{t \bar{t}}$, the conversion factor from MC mass to pole mass would experimentally turn out to be constant within uncertainties. This is not completely obvious, since the MC mass is mainly evaluated from kinematical quantities within each event, while the pole mass is evaluated from the derived cross sections. Here, we will simply make the assumption that the ratio is constant, and neglect the related (currently unknown) additional uncertainties, which is the weakest point of the procedure.

In a first step, we use this constant conversion factor to translate the $m_{t t}$-scale-dependent MCmass evaluation [43] into a corresponding scale-dependent evaluation of the top pole mass. In the much better defined second step, we translate [39] the top pole mass (semi)independently obtained at each scale $m_{t \bar{t}}$ into the corresponding $\overline{M S}$ running mass $m_{t}(\mu)$ with $\mu=m_{t \bar{t}}$. Somewhat arbitrarily, we use the two-loop formula. In the final step we again use Eq. (1) to translate each mass into a Yukawa coupling.

The result of this preliminary procedure is shown in Fig. 2, together with various evaluations of $\alpha_{s}$ at different scales. The blue bands showing the running expected from Standard Model theory are obtained by using the PDG reference values [38] for each of the parameters with their uncertainties, and evolving them using the three-loop QCD formula for $\alpha_{s}$, the one-loop QCD formula for the charm and Higgs Yukawa couplings, and the two-loop formula for top, consistent with the way the points have been extracted from the data. No Higgs or electroweak loop corrections are applied. The Yukawa couplings from direct measurements [40,41] are also shown in the figure, appropriately evaluated at the scale $m_{H}$.

Although not yet quite perfect (see some of the caveats explained above) this is, to the best knowledge of the author, the first graphical representation of the running of Higgs Yukawa couplings extracted from data. It is shown together with the well-known running of $\alpha_{s}$ extracted from $\tau$ decays [38], Upsilon decays [49], PETRA [50], LEP [51], HERA [52], S $p \bar{p}$ S [53], Tevatron [54] and LHC $[45,55]$ data to illustrate the analogy. It will be noted that data and Standard Model expectations are consistent, and that the couplings extracted indirectly from the mass measurements are consistent with those extracted from direct measurements of Higgs production. This nicely illustrates the interplay between QCD and the Higgs sector in the Standard Model.

\section{Conclusions}

The most recent measurements of charm production at HERA have been presented and put into the context of other measurements. With these examples, the author hopes to have illustrated that, if the interpretation of such measurements is pursued all the way down to the basics of the Standard Model, heavy quark physics (including charm physics) is almost always also both QCD and Higgs physics, since the quark masses almost always play a role. So far, the Higgs Yukawa 


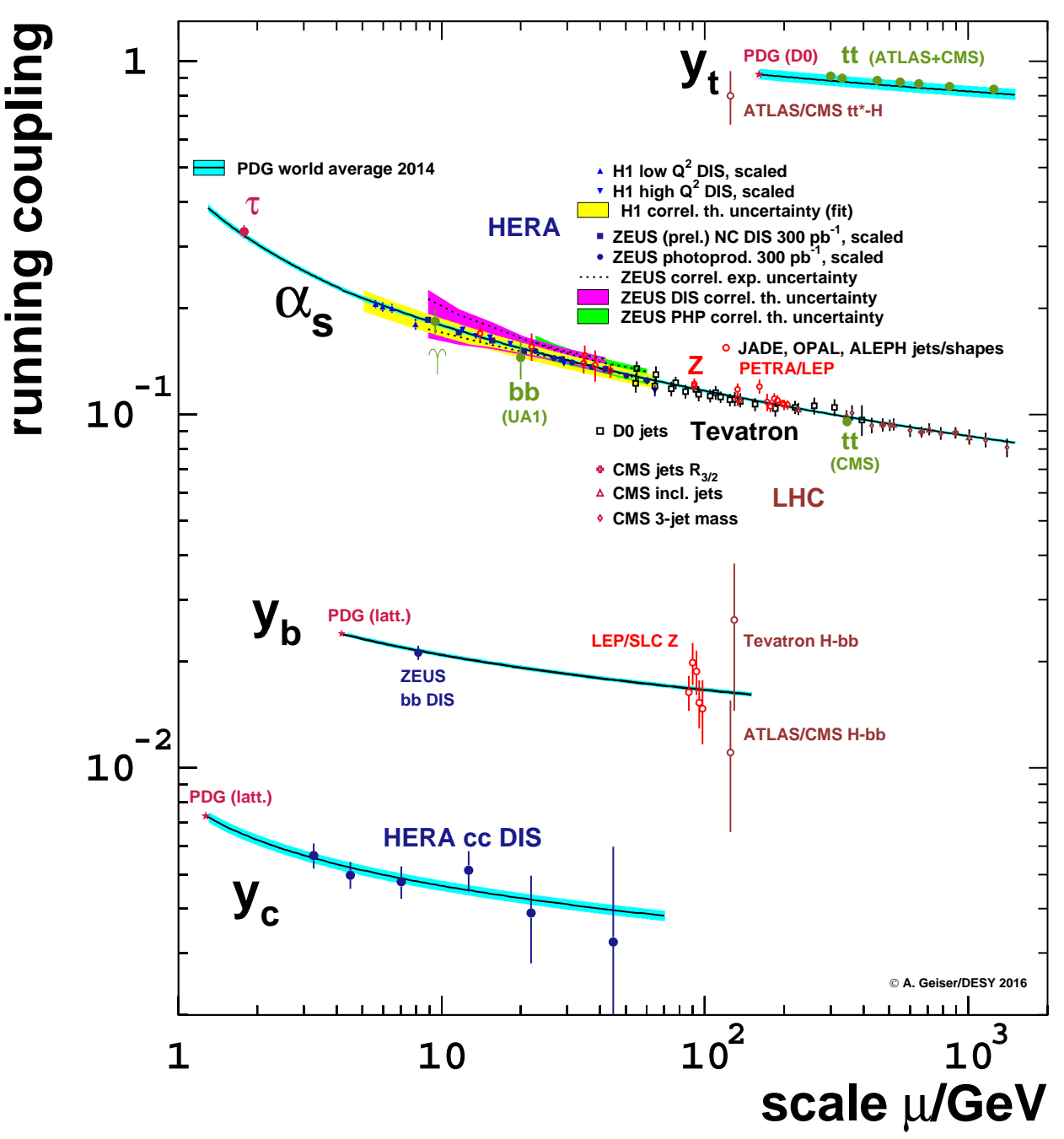

Figure 2: The running of the Higgs Yukawa couplings for charm $\left(y_{c}\right)$, beauty $\left(y_{b}\right)$ and top $\left(y_{t}\right)$ as a function of energy/momentum scale $\mu$, compared to the running of QCD coupling constant $\alpha_{s}$. For references, details of their evaluation and the scheme definitions see text. The brown points at scale $m_{H}$ are directly extracted Higgs couplings, while all other Yukawa coupling points are obtained indrectly from mass measurements. The red stars are the PDG world averages, and the blue bands represent their expected evolution.

couplings and their running as obtained from measurements of the quark masses are consistent with the Yukawa couplings obtained from direct measurements of processes involving Higgs bosons.

\section{Acknowledgements}

The speaker is deeply endebted to the ZEUS, H1, PROSA, and xFitter developer collaborations in the context of which most of the results presented in sections 2-4 were obtained. Discussions with V. Bertone on parts of this writeup are gratefully acknowledged. 


\section{References}

[1] O. Behnke, A. Geiser and M. Lisovyi, Charm, Beauty and Top at HERA, Prog. Part. Nucl. Phys. 84 (2015) 1 [arXiv:1506.07519].

[2] O. Zenaiev, Charm Production and QCD Analysis at HERA and LHC, arXiv:1612.02371, to be published.

[3] N. Brambilla et al., Heavy Quarkonium: progress, puzzles, and opportunities, Eur. Phys. J. C71 (2011) 1534 [arXiv:1010.5827].

[4] G. Wolf, Review of High Energy Diffraction in Real and Virtual Photon Proton scattering at HERA, Rept. Prog. Phys. 73 (2010) 116202 [arXiv:0907.1217].

[5] H1 and ZEUS Coll., H. Abramowicz et al., Combination of differential $D^{*}$ cross-section measurements in deep-inelastic ep scattering at HERA, JHEP 1509 (2015) 149 [arXiv:1503.06042].

[6] H1 and ZEUS Coll., F.D. Aaron et al., Combination and QCD Analysis of Charm Production Cross Section Measurements in Deep-Inelastic ep Scattering at HERA, Eur. Phys. J. C73 (2013) 2311 [arXiv:1211.1182].

[7] M. Lisovyi, A. Verbytskyi, O. Zenaiev, Combined analysis of charm-quark fragmentation-fraction measurements, Eur. Phys. J. C76 (2016) 397 [arXiv:1509.01061]; and references therein.

[8] ZEUS Coll., H. Abramowicz et al., Measurement of charm fragmentation fractions in photoproduction at HERA, JHEP 1309 (2013) 058 [arXiv:1306.4862].

[9] ZEUS Coll., H. Abramowicz et al., Measurement of the cross-section ratio $\sigma_{\psi(2 S)} / \sigma_{J / \psi(1 S)}$ in deep inelastic exclusive ep scattering at HERA, Nucl. Phys. B909 (2016) 934 [arXiv:1606.08652]; and references therein.

[10] H1 Coll., C. Adloff et al., Charmonium production in deep inelastic scattering at HERA, Eur. Phys. J. C10, 373 (1999) [hep-ex/9903008].

[11] H1 Coll., C. Adloff et al., Photoproduction of psi (2S) mesons at HERA, Phys. Lett. B421, 385 (1998) [hep-ex/9711012].

[12] H1 and ZEUS Coll., H. Abramowicz et al., Combination of measurements of inclusive deep inelastic $e^{ \pm} p$ scattering cross sections and QCD analysis of HERA data, Eur. Phys. J. C75 (2015) 580 [arXiv:1506.06042].

[13] MMHT Coll., L.A. Harland-Lang et al., Charm and beauty quark masses in the MMHT2014 global PDF analysis, Eur. Phys. J. C76 (2016) 10 [arXiv:1510.02332];

MMHT Coll., L.A. Harland-Lang et al., Parton distributions in the LHC era: MMHT 2014 PDFs, Eur. Phys. J. C75 (2015) 204 [arXiv:1412.3989].

[14] CTEQ Coll., S. Dulat et al., New parton distribution functions from a global analysis of quantum chromodynamics, Phys. Rev. D93 (2016) 033006 [arXiv:1506.07443];

J. Gao et al., CT10 next-to-next-to-leading order global analysis of QCD, Phys. Rev. D89 (2014) 033009 [arXiv:1302.6246].

[15] NNPDF Coll., R.D. Ball et al., Parton distributions for the LHC Run II, JHEP 1504 (2015) 040 [arXiv:1410.8849].

[16] P. Jimenez-Delgado, E. Reya, Delineating parton distributions and the strong coupling, Phys. Rev. D89 (2014) 074049 [arXiv:1403.1852]. 
[17] ABM Coll., S. Alekhin, J. Blümlein and S. Moch, The ABM parton distributions tuned to LHC data, Phys. Rev. D89 (2014) 054028 [arXiv:1310.3059].

[18] PROSA Collaboration, O. Zenaiev et al., Impact of heavy-flavour production cross sections measured by the LHCb experiment on parton distribution functions at low x, Eur. Phys. J. C75 (2015) 396 [arXiv:1503.04581].

[19] LHCb Collaboration, R. Aaij et al., Prompt charm production in pp collisions at $\sqrt{s}=7 \mathrm{TeV}$, Nucl. Phys. B871 (2013) 1 [arXiv:1302.2864].

[20] ZEUS Coll., H. Abramowicz et al., Measurement of beauty and charm production in deep inelastic scattering at HERA and measurement of the beauty-quark mass, JHEP 1409 (2014) 127 [arXiv:1405.6915].

[21] LHCb collaboration, R. Aaij et al., Measurement of B meson production cross-sections in proton-proton collisions at $\sqrt{s}=7 \mathrm{TeV}$, JHEP 1308 (2013) 117 [arXiv:1306.3663].

[22] R. Gauld et al., Charm production in the forward region: constraints on the small-x gluon and backgrounds for neutrino astronomy, JHEP 1511 (2015) 009, [arXiv:1506.08025].

[23] R. Gauld and J. Rojo, Precision determination of the small-X gluon from charm production at LHCb, arXiv:1610.09373, to be published.

[24] M.V. Garzelli, S. Moch and G. Sigl, Lepton fluxes from atmospheric charm revisited, JHEP 1510 (2015) 115, [arXiv:1507.01570].

[25] PROSA Collaboration, M.V. Garzelli et al., Prompt neutrino fluxes in the atmosphere with PROSA parton distribution functions, arXiv:1611.03815, to be published.

[26] IceCube Collaboration, M. Aartsen et al., Evidence for High-Energy Extraterrestrial Neutrinos at the IceCube Detector, Science 342 (2013) 1242856 [arXiv:1311.5238].

[27] S. Alekhin et al., Determination of the charm-quark mass in the $\overline{M S}$ scheme using charm production data from deep inelastic scattering at HERA, Phys. Lett. B718 (2012) 550 [arXiv:1209.0436].

[28] S. Alekhin et al., Precise charm-quark mass from deep-inelastic scattering, Phys. Lett. B720 (2013) 172 [arXiv:1212.2355].

[29] xFitter Developers' Team, V. Bertone et al., A determination of $m_{c}\left(m_{c}\right)$ from HERA data using a matched heavy-flavor scheme, JHEP 1608 (2016) 050 [arXiv:1605.01946].

[30] S. Alekhin and S. Moch, Heavy-quark deep-inelastic scattering with a running mass, Phys. Lett. B699 (2011) 345 [arXiv:1011.5790].

[31] J. Gao, M. Guzzi and P.M. Nadolsky, Charm quark mass dependence in a global QCD analysis, Eur. Phys. J. C73 (2013) 2541 [arXiv:1304.3494].

[32] S. Alekhin, J. Blümlein and S. Moch, Determination of $\alpha_{s}$ and $m_{c}$ in deep-inelastic scattering, Mod. Phys. Lett. A28 (2013) 1360018 [arXiv:1307.1219].

[33] S. Moch, Precise heavy-quark masses, Nucl. Part. Phys. Proc. 261-262 (2015) 130; and references therein.

[34] S. Alekhin et al., PDFs, $\alpha_{s}$ and quark masses from the global fits, PoS LL2016 (2016) 042, arXiv:1609.07417;

S. Alekhin et al., Parton Distribution Functions, $\alpha_{s}$ and Heavy-Quark Masses for LHC Run II, arXiv:1701.05838. 
[35] Particle Data Group, C. Patrignani et al., The Review of Particle Physics (2016), Chin. Phys. C40 (2016) 100001 [http://www-pdg.lbl.gov/].

[36] H1 and ZEUS Collaborations and S. Moch, Determination of Charm Mass Running from an Analysis of Combined HERA Charm data, H1-prelim-14-071, ZEUS-prel-14-006;

A. Gizhko, Proceedings of 2014 International Workshop on Diffraction in High-Energy Physics, Primosten, Croatia, September 10-16 2014.

[37] A. Gizhko, PhD thesis, Measurement of beauty quark mass at HERA and impact on Higgs production in association with beauty quarks at LHC, Hamburg University 2016, DESY-THESIS-2016-015, CERN-THESIS-2016-075.

[38] Particle Data Group, K.A. Olive et al., Review of Particle Physics (2014), Chin. Phys. C38 (2014) 090001 [http://pdg.lbl.gov].

[39] B. Schmidt, M. Steinhauser, CRunDec: a C++ package for running and decoupling of the strong coupling and quark masses, Comput. Phys. Commun. 183 (2012) 1845 [arXiv:1201.6149]; K.G. Chetyrkin, J.H. Kühn, M. Steinhauser, RunDec: a Mathematica package for running and decoupling of the strong coupling and quark masses, Comput. Phys. Commun. 133 (2000) 43 [hep-ph/0004189].

[40] ATLAS and CMS Coll., G. Aad et al., Measurements of the Higgs boson production and decay rates and constraints on its couplings from a combined ATLAS and CMS analysis of the LHC pp collision data at $\sqrt{s}=7$ and $8 \mathrm{TeV}$, JHEP 1608 (2016) 045 [arXiv:1606.02266].

[41] CDF and D0 Coll., T. Aaltonen et al., Tevatron Constraints on Models of the Higgs Boson with Exotic Spin and Parity Using Decays to Bottom-Antibottom Quark Pairs, Phys. Rev. Lett. 114 (2015) 151802 [arXiv:1502.00967].

[42] DELPHI Coll., J. Abdallah et al., Study of b-quark mass effects in multijet topologies with the DELPHI detector at LEP, Eur. Phys. J. C55 (2008) 525 [arXiv:0804.3883]; and references therein.

[43] CMS Coll., V. Khachatryan et al., Measurement of the top quark mass using proton-proton data at $\sqrt{s}$ $=7$ and 8 TeV, Phys. Rev. D93 (2016) 072004 [arXiv:1509.04044].

[44] ATLAS Coll., G. Aad et al., Determination of the top-quark pole mass using $t \bar{t}+1$-jet events collected with the ATLAS experiment in $7 \mathrm{TeV}$ pp collisions, JHEP 1510 (2015) 121 [arXiv:1507.01769].

[45] CMS Coll., S. Chatrchyan et al., Determination of the top-quark pole mass and strong coupling constant from the $t \bar{t}$ production cross section in pp collisions at $\sqrt{s}=7 \mathrm{TeV}$, Phys. Lett. B728 (2014) 496; Erratum: Phys. Lett. B738 (2014) 526; [arXiv:1307.1907].

[46] CMS Coll., V. Khachatryan et al., Measurement of the $t \bar{t}$ production cross section in the e- $\mu$ channel in proton-proton collisions at sqrt(s) = 7 and 8 TeV, JHEP 1608 (2016) 029 [arXiv:1603.02303].

[47] CMS Coll., V. Khachatryan et al., Measurement of the differential cross section for top quark pair production in pp collisions at $\sqrt{s}=8 \mathrm{TeV}$, Eur. Phys. J. C75 (2015) 542 [arXiv:1505.04480].

[48] LHC top working group, ATLAS, CMS and theory; see plots at https://twiki.cern.ch/twiki/bin/view/LHCPhysics/LHCTopWGSummaryPlots

[49] N. Brambilla et al., Extraction of $\alpha_{s}$ from radiative Upsilon(1S) decays, Phys. Rev. D75 (2007) 074014 [hep-ph/0702079]. 
[50] JADE Coll., J. Schieck et al., Measurement of the strong coupling $\alpha_{s}$ from the three-jet rate in $e^{+} e^{-}$-annihilation using JADE data, Eur. Phys. J. C73 (2013) 2332 [arXiv:1205.3714];

JADE Coll., S. Bethke et al., Determination of the Strong Coupling $\alpha_{s}$ from hadronic Event Shapes with $O\left(\alpha_{s}^{3}\right)$ and resummed QCD predictions using JADE Data, Eur. Phys. J. C64 (2009) 351 [arXiv:0810.1389].

[51] OPAL Coll., G. Abbiendi et al., Determination of $\alpha_{s}$ using OPAL hadronic event shapes at $\sqrt{s}=91$ $209 \mathrm{GeV}$ and resummed NNLO calculations, Eur. Phys. J. C71 (2011) 1733 [arXiv:1101.1470]; G. Dissertori et al., Determination of the strong coupling constant using matched NNLO+NLLA predictions for hadronic event shapes in $e^{+} e^{-}$annihilations, JHEP 0908 (2009) 036 [arXiv:0906.3436].

[52] ZEUS Coll., H. Abramowicz et al., Inclusive-jet photoproduction at HERA and determination of $\alpha_{s}$, Nucl. Phys. B864 (2012) 1 [arXiv:1205.6153];

H1 Coll., F.D. Aaron et al., Jet Production in ep Collisions at Low $Q^{2}$ and Determination of $\alpha_{s}$, Eur. Phys. J. C67 (2010) 1 [arXiv:0911.5678];

H1 Coll., F.D. Aaron et al., Jet Production in ep Collisions at High $Q^{2}$ and Determination of $\alpha_{s}$, Eur. Phys. J. C65 (2010) 363 [arXiv:0904.3870];

ZEUS Collaboration, Inclusive-jet production in NC DIS with HERA II, ZEUS-prel-10-002 (2010).

[53] UA1 Coll., C. Albajar et al., Measurement of $\alpha_{s}$ from $b \bar{b}$ production at the CERN $p \bar{p}$ collider, Phys. Lett. B369 (1996) 46-54 [CERN-PPE-95-158].

[54] D0 Coll., V.M. Abazov et al., Determination of the strong coupling constant from the inclusive jet cross section in $p \bar{p}$ collisions at $\sqrt{s}=1.96 \mathrm{TeV}$, Phys. Rev. D80 (2009) 111107 [arXiv:0911.2710]; D0 Coll., V. Mukhamedovich et al., Measurement of angular correlations of jets at $\sqrt{s}=1.96 \mathrm{TeV}$ and determination of the strong coupling at high momentum transfers, Phys. Lett. B718 (2012) 56 [arXiv:1207.4957].

[55] B. Malaescu, P. Starovoitov, Evaluation of the Strong Coupling Constant $\alpha_{s}$ Using the ATLAS Inclusive Jet Cross-Section Data, Eur. Phys. J. C72 (2012) 2041 [arXiv:1203.5416];

CMS Coll., S. Chatrchyan et al., Measurement of the ratio of the inclusive 3-jet cross section to the inclusive 2-jet cross section in pp collisions at $\sqrt{s}=7 \mathrm{TeV}$ and first determination of the strong coupling constant in the TeV range, Eur. Phys. J. C73 (2013) 2604 [arXiv:1304.7498]; CMS Coll., V. Khachatryan et al., Measurement of the inclusive 3-jet production differential cross section in pp collisions at $7 \mathrm{TeV}$ and determination of the strong coupling constant in the TeV range, Eur. Phys. J. C75 (2015) 186 [arXiv:1412.1633];

CMS Coll., V. Khachatryan et al., Constraints on parton distribution functions and extraction of the strong coupling constant from the inclusive jet cross section in pp collisions at $\sqrt{s}=7 \mathrm{TeV}$, Eur. Phys. J. C75 (2015) 288 [arXiv:1410.6765]. 